\title{
Improvement of gear pitch machining accuracy based on the principle of phase-transfer gear-grinding technique
}

\author{
Siying Ling ${ }^{1,2^{*}}$, Kun Wang ${ }^{1}$, Baodi Yu ${ }^{1}$, Xiaodong Wang ${ }^{1,2}$, Liding Wang ${ }^{1,2}$ \\ 1.Key Laboratory for Precision \& Non-traditional Machining of Ministry of Education, Dalian \\ University of Technology, Dalian 116023,
}

2. Key Laboratory for Micro/Nano Technology and System of Liaoning Province, Dalian University of Technology, Dalian 116023,

*Corresponding author, E-mail:luckling168@163.com

\section{Key words: Phase transfer,Indexing error,Pitch deviation,Gear grinding Technology}

Abstract: In order to improve the pitch machining accuracy of gear artefacts, based on the extraction method of the systematic indexing error (SIE), the phase-transfer gear-grinding technique (PGT) was proposed by taking the SIE curve interfere with its phase-transfer error curve. First, the SIE curve of the gear grinding machine (GGM) was precisely extracted by adopting the 36 polygon mirror which was precisely calibrated by using the closure measurement method and the autocollimator, and the maximum indexing error is obtained as 6.82". Then the characteristics and the phase-transfer strategies to eliminate the main harmonic component of the SIE curve of the GGM was analysed. Finally, precision grinding experiments were carried out by adopting the traditional gear-grinding technique (TGT) and the PGT. The experiments show that, the total cumulative pitch deviation of gear specimen is obtained as $2.57 \mu \mathrm{m}$ by adopting the TGT, and the relative error is increased approximately by $24 \%$ comparing with the SIE. However, the total cumulative pitch deviation of gear specimen is obtained as $1.34 \mu \mathrm{m}$ by adopting the PGT, and the relative error is reduced approximately by $36 \%$ comparing with the SIE. The results show that the pitch machining accuracy of the gear specimen is significantly improved and is higher than the indexing accuracy of the GGM by adopting PGT, and the pitch deviations of the gear specimen achieve the highest class of the current gear standards. The researches have important guiding significance for the development of ultra-precision indexing components, including gear artefacts, indexing plates, end-tooth plates, indexing worm gears, the harmonic gears and so on.

\section{Abbreviations}

$\mathrm{CMM}=$ Coordinate measuring machines

GBA $=$ Gauge block gear-like artefact

GGM=Gear grinding machines

GMI $=$ Gear measuring instruments

MBA = Multi-ball artefact
NTT $=$ Neighbor-tooth translocation technique

OCT $=$ Offset-compensated technique

PGT $=$ Phase-transfer gear-grinding technique

$\mathrm{SIE}=$ Systematic indexing error

TGT $=$ Traditional gear-grinding technique

\section{Introduction}

Pitch deviations are default inspection items of national standard GB/T 10095.1-2008 and international standard ISO 1328-1:2013(E) of cylindrical gears, ${ }^{1,2}$ also are the classification basis of the accuracy class of gear artefacts according to JJG 1008-2006. ${ }^{3}$ Effect of pitch deviations on gear 
vibration and noise has increased at micron level; its value has reached the highest class of the gear national standard and international standard. To achieve such precision measurement on gear pitch deviations, it is necessary to have high precision gear measurement instruments (GMI), ${ }^{4}$ high precision measurement methods and high precision gear artefacts. High precision artefacts are used to calibrated GMI, to ensure measurement traceability of the gear pitch deviations. ${ }^{5}$ In addition, the high precision measurement method can further separate the SIE of GMI, to improve the measurement accuracy. ${ }^{9}{ }^{6}$ Since the manufacture of the high precision gear artefact is very difficult, the existing precision gear artefact products cannot achieve the precision calibration of the high-precision gear pitch measuring instruments.

After calibrated by high-precision gear artefact, GMI and the coordinate measuring machine (CMM) have the ability to measure gear pitch deviations with class 1 accuracy. Since the highest manufacture accuracy of the current commercial gear pitch artefacts is only class $2 \sim 1$, they cannot achieve the high-precision calibration of a GMI. For this, on the calibration of the GMI and the evaluation of measurement uncertainty, some scholars adopted gear-like pitch artefacts to calibrate GMI. Maria Pia Sammartini from Italy designed gauge block artefact (GBA) used to evaluate measurement uncertainty of GMI. For the lower assembly accuracy of GBA, which the single pitch deviation is only $19 \mu \mathrm{m}$ and the total cumulative pitch deviation is only $45 \mu \mathrm{m}$, it is not fit to calibrate a high-precision GMI. Yohan Kondo from Japan designed multi-ball pitch artefact (MBA) to calibrate GMI and evaluate the measurement uncertainty. The locating accuracy after assembly main depends on the manufacture accuracy of the locating holes. Though the accuracy of ball and gauge can achieved very high, however it is difficult to manufacture high precision locating holes, so the accuracy of gear-kind pitch artefacts are hard to achieve high precision. Based on MBA, Japanese scholars proposed a method to set a number of high precision steel balls arrange around a high-precision cylinder by adopting magnetic force or spring force, which solved the MBA locating problem. ${ }^{7}$ Though the improved MBA has high precision and is easy to assemble, however, the number of balls is limited, so it is difficult to achieve the precision calibration of a GMI. Besides, the structural rigidity, the location repeatability and the traceability reliability of such kind of artefacts needs further studies.

Compared with the gear-like artefacts(GBA and MBA), high precision gear artefacts after adequate heat treatment and ultra-precision machining have many advantages of structural rigidity, dimensional stability and high precision on the aspect of calibrating the GMI and indexing datum transfer. However, raising the pitch manufacture accuracy of the gear artefact to micron level is still a huge challenge.

Ling SY have proposed two gear-grinding techniques to improve pitch deviations of ultra-precision gear. Based on the offset-compensated technique (OCT), the neighbour-tooth translocation technique (NTT) was proposed to improve the single pitch deviation of cylindrical gears. However, there is a certain space to improve the machining accuracy of the total cumulative pitch deviation by making innovations of the gear-grinding technique.

According to the law of error transfer, the error will gradually increase during the transfer process. In other words, the pitch machining accuracy of the gear to be ground is generally less than the indexing accuracy of a machine tool. To manufacture a high-precision gear pitch artefact requires the indexing system with the higher indexing accuracy. However, as for ultra-precision machining of gear artefacts at micron level, there is not enough space by selecting the indexing device with the higher indexing accuracy to improve the gear pitch machining accuracy.

If that the machining accuracy of gear pitch is higher than the accuracy of indexing device of the 
GGM can be achieved through the innovations of the gear-grinding technique, the ultra-high machining of the indexing elements will be achieved. Based on this idea, the PGT was proposed by taking the SIE curve interfere with its phase-transfer error curve.

\section{The extraction of the SIE}

There are a variety of methods to extract the SIE curve of the GGM. According to the experimental conditions, this article selects the autocollimator and 36 polygon mirror to extract 36 uniform discrete points of the SIE of the GGM. Since the SIE of the GGM and the 36 polygon mirror what is adopted in the experiment are in the same order $\left(4^{\prime \prime} \sim 8^{\prime \prime}\right)$, it is necessary to calibrate the SIE of 36 polygon mirror precisely to improve its measurement accuracy by closure measurement method. Then, the 36 polygon mirror after calibrating precisely and relative measurement method are adopted to extract the SIE curve of the GGM Y7125. The calibration of 36 polygon mirror is shown in Figure 1. Extraction of the SIE of the GGM is done by adopting the calibrated 36 polygon mirror and the relative method as shown in Figure2.

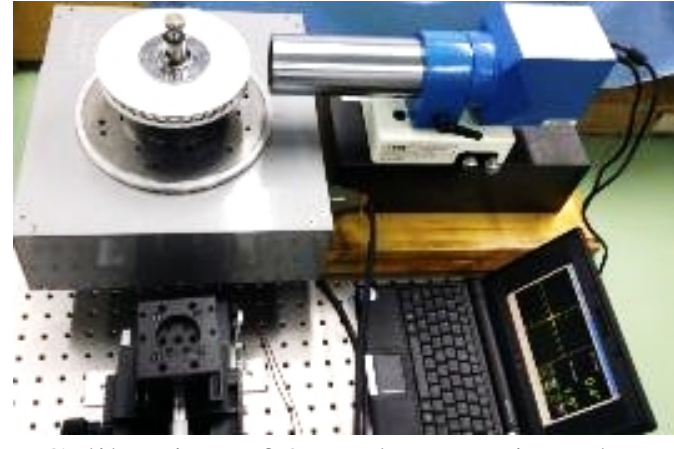

Figure 1 Calibration of 36 polygon mirror by closure measurement method

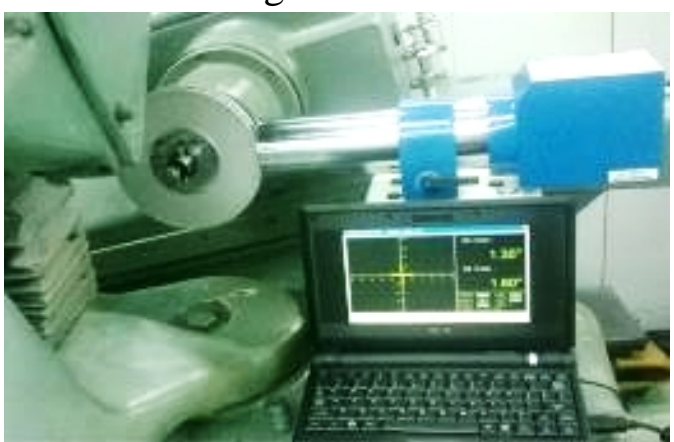

Figure 2 Extraction of the SIE of the GGM

The SIE curve $E_{0}$ consisting of 36 uniform discrete points of the GGM is shown in Figure 3. As can be seen from the cure $E_{0}$, the maximum indexing error of the GGM is 6.82 ".

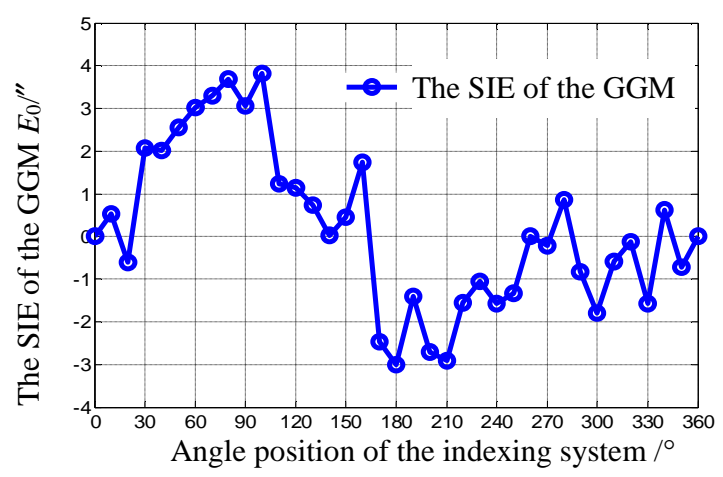

Figure 3 The SIE curve of the GGM

The SIE of the GGM is one of the main error source of gear pitch deviations.

\section{Characteristics of the SIE curve}

The each order of harmonic component can be isolated through the discrete data trigonometric Fourier transform of SIE of the GGM. For convenience, the low-order harmonic component and residual error of the SIE curve of the GGM is isolated through the method of finite order curve fitting of the trigonometric Fourier series. The equation of the fitting curve is: 


$$
f(x)=a_{0}+\sum_{n=1}^{k} a_{n} \sin \left[\frac{n \pi}{180}\left(x-\varphi_{n}\right)\right]
$$

where $a_{0}$ is the average of 36 uniform discrete points of the SIE of the GGM; $a_{\mathrm{n}}$ is the amplitude of $n$-order harmonic component, $n \pi / 180$ is the frequency of the harmonic component; $\varphi_{\mathrm{n}}$ is the initial phase angle of $n$-order harmonic component and $k$ is the maximum order of the curve fitting.

Curve fitting is conducted by using CFTOOl curve fitting toolbox of Matlab with the maximum order $k=6$, and $a_{0}$ is calculated by the following equation:

$$
a_{0}=\sum_{n=1}^{36} \frac{E_{0}\left(x_{n}\right)}{n}
$$

The amplitudes and their initial phase angle of 1 6 order harmonic component are listed in Table 1 after curve fitting of the SIE curve of the GGM.

Table 1 Amplitude and initial phase angle of 1 6 order harmonic component

\begin{tabular}{ccccccc}
\hline$n$ & 1 & 2 & 3 & 4 & 5 & 6 \\
\hline$a_{n}{ }^{\prime \prime}$ & 2.07 & 1.19 & 0.19 & 0.40 & 0.16 & 0.44 \\
\hline$\varphi_{n}{ }^{\circ}$ & -26.0 & 42.7 & 5.6 & -130.3 & -15.1 & 20.2 \\
\hline
\end{tabular}

The 2-order harmonic component of the SIE curve of the GGM is mainly caused by the installation swing error of the indexing plate relative to the gear grinding machining spindle. If the installation of the indexing plate is swung, the projection on the normal plate of grinding machine spindle of reference circle of the indexing plate is turned into the ellipse, which results in the 2-order harmonic component in the SIE curve of the GGM. In addition, the machining error of the indexing plate also contains a certain amplitude of the 2-order harmonic component.

\section{The principle of PGT}

According to the characteristics of the harmonic curve, superposition of the harmonic curves with same frequency and phase difference of half a cycle can reduce the amplitude of harmonic component to the most extent. As to the odd order harmonic components, the phase difference is $180^{\circ}$; as to the even order harmonic components, the phase difference is $90^{\circ}$.

The amplitude of the harmonic component can be reduce by applying the principle mentioned above to the gear machining process. The second gear-grinding process is conducted by changing the installation position between gear to be ground and the indexing plate. With the principle of the SIE curve interfering with its phase-transfer curve, the gear tooth thickness of some gears with a larger machining allowance is further reduced, and some teeth without machining allowance are not involved in the second gear-grinding, thus the accuracy of the gear pitch machining is improved. Taking the basic harmonic component as an example, the principle of the PGT is further described, as shown in Figure 4. 


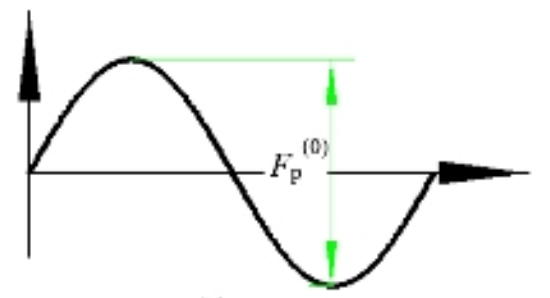

(a)

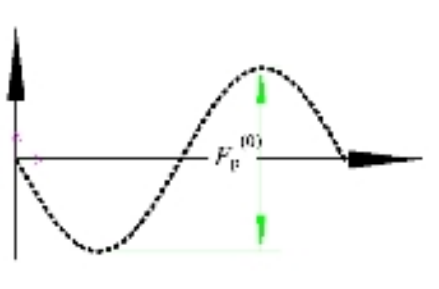

(b)

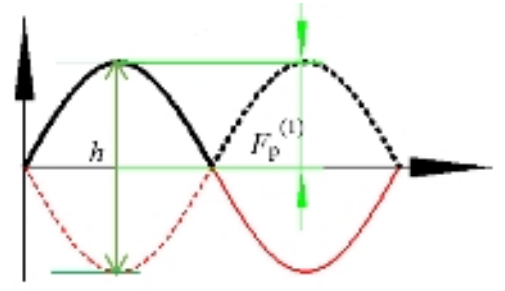

(c)

(a) The basic harmonic component in the SIE curve,

(b) The harmonic component after phase transferring at $180^{\circ}$,

(c) The interference curve between curve (a) and curve (b).

Figure 4 Principle of the PGT

The interference depth $h$ of the two curve can be controlled by adjusting the feed amount of the grinding wheel during the second gear-grinding process. As can be seen from Figure 4, when the interference depth $h$ is equal to two times of the peak-valley value of the harmonic component, the peak-valley value of the superposition error curve is minimum, which can reaches half of the amplitude of the original curve. At this condition, the interference depth $h$ is called the best interference depth.

\section{Gear grinding experiment}

According to the condition of the laboratory, the precision gear grinding experiment as shown in Figure 5 is conducted by selecting the parameters of the gear specimen as shown in Table 2.

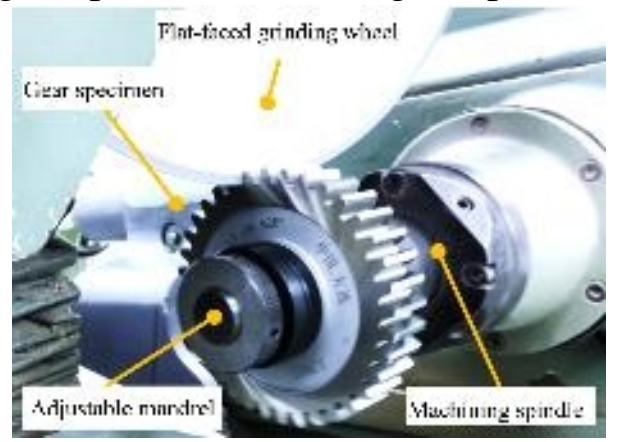

Figure 5 Gear grinding experiment

Table 2 Parameters of gear specimen

\begin{tabular}{ccccc}
\hline $\begin{array}{c}\text { Module } \\
m / \mathrm{mm}\end{array}$ & $\begin{array}{c}\text { Number of teeth } \\
z\end{array}$ & $\begin{array}{c}\text { Pressure angle } \\
\alpha /{ }^{\circ}\end{array}$ & $\begin{array}{c}\text { Helix angle } \\
\beta /{ }^{\circ}\end{array}$ & $\begin{array}{c}\text { Facewidth } \\
\mathrm{b} / \mathrm{mm}\end{array}$ \\
\hline 3.5 & 36 & 20 & 0 & 25 \\
\hline
\end{tabular}

Theoretically, the SIE of the GGM will transfer to the gear machining at the same value. Based on studying the indexing error transfer law of the GGM by comparing the difference between the machining accuracy of the gear pitch and the indexing accuracy of the GGM, the PGT is studied. The interference depth $h$ is the sum of the odd order harmonic components and its peak-valley value is 4.84 " (as converting into the displacement at reference circle of gear specimen, the value is 1.48 $\mu \mathrm{m})$.

When the PGT is conducted, the interference depth $h$ of the two curve has the greatest influence on the amplitude of the superimposed curve. We can control the interference depth $h$ of two curves during the second gear grinding process by controlling the feed amount $f$ of the grinding wheel. The 
relationship between them is:

$$
f=h \cos \alpha
$$

where $\alpha$ is the pressure angle of the gear, as to the standard gear, $\alpha=20^{\circ}$.

Based on the above analysis, the each times of the PGT can only reduce the odd order harmonic components or even order harmonic components in the SIE curve. For the odd order harmonic components of the SIE curve of the GGM accounts for a large proportion, so the phase-transfer angle as $180^{\circ}$ is chosen. The feed amount $f$ of the grinding wheel is $1.39 \mu \mathrm{m}$ during the second gear grinding process. Due to the feed resolution of the GGM Y7125 adopted in the study is only $2 \mu \mathrm{m}$, so it is difficult to control the feed amount of the grinding wheel to micrometer. During the actual operation, we can make approximate judgement of the feed amount $f$ according to the number of teeth participated in the second grinding process. The phase-transfer principle diagram shows that when the interference depth $h$ reaches the best, the feed amount of the grinding wheel is about 1.39 $\mu \mathrm{m}$ and about half of the gear teeth will participate in the second grinding process. Due to the differences between the profile slope deviation and the helix slope deviation of each tooth, there is local contact in tooth root, tooth top, left side or right side of the tooth surface. When we adopt the PGT, those gear teeth with local contact are not included the total number of teeth in grinding. Only when the whole tooth surface of the gear participates in the second grinding process uniformly, it can be included in the total number of teeth. The continuous feeding to submicron can be achieved by exploiting the thermal elongation of the grinding wheel spindle. The second grinding process was conducted at about 40 minutes before the grinding wheel spindle to the thermal equilibrium, and the number of teeth participated in the second grinding process was controlled by thermal elongation of grinding wheel spindle and rotating handwheel of the feed system to micro level. Due to the phenomenon of error reflection and the existence of the elastic deformation of grinding system, the amount of the actual thinning of tooth surface during grinding process is less than the feed amount of the grinding wheel. So we are appropriate to increase the number of teeth participation in the second grinding process to achieve the best effect of the PGT. According to the performance parameters of the GGM and the grinding experience, it can be seen that when the number of teeth of the second grinding process reaches $2 / 3$ to $3 / 4$ of the total number of teeth, the grinding effect is the best. In this experiment, by controlling the number of teeth participated in the second grinding process from 24 to 27 , the smaller total cumulative pitch deviation of the gear specimens can be obtained.

In $20 \pm 1^{\circ} \mathrm{C}$ constant temperature room, the ultra-precision pitch measuring device which we designed is adopted to test the gear pitch deviation of the gear specimen. The comparison between the individual pitch cumulative deviation $\left(F_{\mathrm{pi}}\right)$ of the specimen by adopting the TGT and the PGT and the SIE of the GGM (for comparison convenience, the second of angle " is converted into the displacement at reference circle $\mu \mathrm{m}$ ) is shown in Figure 6. 


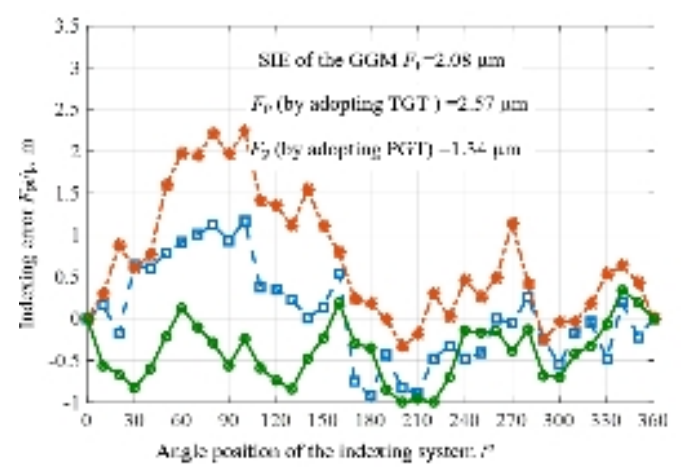

Figure 6 Comparison between PGT and TGT

The Figure 6 shows that by adopting TGT, the cumulative pitch deviation curve of the gear specimen and error curve of the GGM indexing system almost have the same change trend, but there is a tendency to increase. The cumulative indexing error of the GGM at 36 uniform discrete points converts into the displacement at reference circle is $2.08 \mu \mathrm{m}$, while the total cumulative pitch deviation $F_{\mathrm{p}}$ of the gear specimen is $2.57 \mu \mathrm{m}$, and the total cumulative pitch deviation is increased by about $24 \%$. The SIE of the GGM is affected by many uncertain factors during it transmission from the GGM to the gear to be ground, which leads to the increasing tendency of the indexing error. According to the error transfer rules and the SIE of the GGM, we can predict gear pitch accuracy. The total cumulative pitch deviation of gear specimen is reduced to $1.34 \mu \mathrm{m}$ with $U_{95}$ less than $0.6 \mu \mathrm{m}$ by adopting the PGT, and the relative error is reduced approximately by $36 \%$ comparing with that of the SIE. The single pitch deviation is reduced to $0.56 \mu \mathrm{m}$ with $U_{95}$ less than $0.4 \mu \mathrm{m}$, and the relative error is reduced approximately by $54 \%$ comparing with that of the SIE. It is obvious that the gear pitch machining accuracy of the gear specimen is higher than the SIE of the GGM by adopting the PGT proposed in the article.

The highest class of the single pitch deviation and the total cumulative pitch deviation in the current national standard GB/T 10095.1 2008, the international standard ISO 1328-1:2013 (E) and Germany standard DIN 3962-1978, and the measured value of pitch deviations of the gear specimens are shown in Table 3.

Table 3 The highest class of gear pitch deviations and measured value of gear specimen

Pitch tolerance GB/T 10095.1-2008 DIN 3962-1978 ISO 1328-1:2013 (E) Measured value

\begin{tabular}{ccccc} 
& Class 0 & Class 1 & Class 1 & \\
\hline$f_{\mathrm{pT}} / \mu \mathrm{m}$ & $\mathbf{1 . 1}$ & $\mathbf{1 . 5}$ & $\mathbf{1 . 6}$ & $\mathbf{0 . 5 6} \pm \mathbf{0 . 4}$ \\
\hline$F_{\mathrm{pT}} / \mu \mathrm{m}$ & $\mathbf{3 . 4}$ & $\mathbf{5 . 0}$ & $\mathbf{5 . 0}$ & $\mathbf{1 . 3 4} \pm \mathbf{0 . 6}$ \\
\hline
\end{tabular}

Even considered the measurement uncertainty of pitch deviations ${ }^{11}$, the single pitch deviation $f_{\mathrm{p}}$ and the total cumulative pitch deviation $F_{\mathrm{p}}$ have reached the highest class of the Chinese standard (GB/T, Class 0), Germany standard (DIN, Class 1) and the international standard (ISO, Class 1).

\section{Conclusion}

(1) Based on studying the precision extraction method of the SIE of the GGM, the PGT between the SIE curve and its phase-transfer error curve is proposed.

(2) The total cumulative pitch deviation of gear specimen is reduced to1.34 $\mu \mathrm{m}$ with $U_{95}$ less than $0.6 \mu \mathrm{m}$ by adopting the PGT, and the relative error is reduced approximately by $36 \%$ comparing with that of the SIE. The single pitch deviation is reduced to $0.56 \mu \mathrm{m}$ with $U_{95}$ less than $0.4 \mu \mathrm{m}$, and the relative error is reduced approximately by $54 \%$ comparing with that of the SIE. 
(3) By adopting the PTG proposed in the article, the pitch machining accuracy of the gear specimens (indexing precision) is higher than the SIE of the GGM. Even considered the measurement uncertainty of pitch deviations, the single pitch deviation $f_{\mathrm{p}}$ and the total cumulative pitch deviation $F_{\mathrm{p}}$ have reached the highest class of the Chinese standard GB/T 10095.12008 , Germany standard DIN 3962-1978 and the international standard ISO 1328-1:2013 (E) of cylindrical gears.

\section{Acknowledgdments}

This study was supported by the Science Fund for Key Program (U1508211) and for Creative Research Groups (51621064) of National Natural Science Funds of China and the Fundamental Research Funds for the Central Universities (DUT16TD20 and DUT17JC15).

\section{References}

[1] B. Xu, Y. Shimizu, and S. Ito: Pitch deviation measurement of an involute spur gear by a rotary profiling system. J. Precis. Eng. 152-160 (2015), p. 39

[2] P.S. Maria and D.C. Leonardo: Development and validation of a new reference cylindrical gear for pitch measurement. Precis. Eng. 302-309 (2000), p. 24

[3] Y. Kondo, K. Sasajima and S. Osawa: Development of a novel artefact as a reference for gear pitch measuring instruments. J. Manuf. Sci. E: T. ASME. 161-168 (2009), p. 131

[4] M. Komori, F. Takeoka and T. Kiten: Spring-force self-aligned multiball pitch artefact. J. Precis. Eng. 98-109(2016), p. 45

[5] M. Komori, F. Takeoka and T. Kiten: Magnetically self-aligned multiball pitch artefact using geometrically simple features. J. Precis. Eng.160-171(2015), p. 40

[6] K. Kniel, F. Hartig and S. Osawa: Two highly accurate methods for pitch calibration. Meas. Sci. Technol. 110-115 (2009), p. 20 